\title{
Screening for Retinopathy of Prematurity
}

\author{
Claire Hartnett and Michael O'Keefe \\ Children's University Hospital, Temple Street, Dublin 1 \\ Ireland
}

\section{Introduction}

All ophthalmologists and neonatologists are aware that the condition of retinopathy of prematurity is potentially blinding to the premature infant. However it is also one of the few causes of childhood visual disability which is largely preventable. Many extremely preterm infants will develop some degree of retinopathy of prematurity although in the majority this never progresses beyond the level of mild disease which resolves spontaneously without treatment. A small proportion of premature infants develop potentially severe retinopathy which can be detected through retinal screening. If untreated, severe disease can result in serious vision impairment and consequently all babies at risk of sight-threatening retinopathy of prematurity should be screened.

\section{The aim and importance of screening for retinopathy of prematurity}

Retinopathy of prematurity is a proliferative disease of the retinal vasculature and is a potentially blinding disorder of premature infants (Hunter et al. 1992, Gibson et al. 1990). The condition is characterized by proliferation of abnormal fibrovascular tissue at the border of vascularised and non-vascularised retina (Flyn et al. 1977). Timely and accurate screening of this condition is of utmost importance because of the short window of opportunity during which treatment is effective. The 1984 Classification of Retinopathy of Prematurity (An International Classification of Prematurity, 1984) and the 1988 Cryotherapy for Retinopathy of Prematurity (CRYO-ROP) Study (Cryotherapy for retinopathy of prematurity cooperative group. 1988) both confirmed the success of treatment for retinopathy of prematurity and made screening mandatory. Screening has subsequently improved the visual prognosis of this condition. Improved survival of premature infants of gestational age less than 25 weeks gestation and very low birth weight has resulted in an increased incidence of retinopathy of prematurity and an increase in blindness. These factors have significantly increased the importance of screening. Treatment is effective in altering the course of the disease and can prevent blindness. This means that screening infants is the fundamental first step. The Early Treatment for Retinopathy of Prematurity (ETROP) study also confirmed the importance of screening. National based screening guidelines have been based on the varied incidence of disease between countries (Early Treatment of Retinopathy of Prematurity Cooperative Group. 2003). Many developed countries have adopted guidelines and screening criteria based on weight and gestational age and these are modified according to population based studies on the incidence of 
retinopathy of prematurity. There is a varied incidence of the condition in developed countries compared to developing countries (Gilbert et al. 1997). The incidence of retinopathy of prematurity is much higher in developing countries and there is a greater prevalence of the disease in heavier and older premature infants (Phan et al. 2003).

\section{Indications for screening}

Various guidelines for screening of retinopathy of prematurity exist. All guidelines for screening are evidence-based.

\subsection{Screening criteria}

The American Academy of Ophthalmology, the American Academy of Paediatrics and the American Association of Paediatric Ophthalmology and Strabismus have recommended screening infants with birth weights under $1500 \mathrm{~g}$ or gestational age of 30 weeks or less and selected infants with a birth weight between 1500 and $2000 \mathrm{~g}$ or a gestational age greater than 30 weeks with an unstable clinical course who are considered at high risk by their paediatrician or neonatologist (2006). In 2007, after an extensive review of the literature, updated United Kingdom national guidelines recommended retinopathy of prematurity screening for babies of birth weight less than 1501g and / or gestational age less than 32 weeks (up to 31 weeks and six days)(Royal College of Paediatrics \& Child Health \& Royal College of Ophthalmologists. 2007).

\subsection{Screening protocol}

The American guidelines on screening recommend that in babies born up to 27 weeks the first retinopathy of prematurity screen should be performed at 31 weeks post menstrual age. Those born at 28 weeks gestation or older are screened at 4 weeks after birth. The United Kingdom guidelines recommend that babies born before 27 weeks (up to 26 weeks and 6 days) the first retinopathy of prematurity screen should be performed at 30 to 31 weeks postmenstrual age. Those born at 27 to 31 weeks and 6 days gestational age should have their first screen between four to five weeks after birth. It is recommended that repeat examinations should take place every two weeks thereafter. However repeat examinations should take place weekly when the vessels end in zone 1 or posterior zone 2 , there is plus or pre plus disease or there is any stage 3 disease in any zone. Of particular importance is the retinopathy of prematurity examination at about 34 weeks post menstrual age as this is the age at which the risk of severe disease is considered greatest. In countries where resources and personnel for screening are scarce, the 34 week screen may be the only examination to take place and is also labelled as the one stop examination. Although screening for retinopathy in premature babies should follow the above protocol, it is acknowledged that there may be clinical or organisational circumstances which prevent this. Where a decision is made not to screen a baby, the reason for doing so should be clearly stated in the baby's medical record and the examination should be rescheduled within one week of the intended examination.

\subsection{Threshold disease}

The presence of threshold disease signals the need for treatment. Threshold disease is defined by the Cryotherapy for Retinopathy of Prematurity (CRYO-ROP) Study as at least five continuous or eight cumulative clock hours of Stage 3 retinopathy of prematurity in zone I or II with plus disease. There are other definitions of threshold disease in use, for 
example, the broader United Kingdom guidelines which in addition to the above also include five continuous clock hours or eight cumulative clock hours of stage 3 retinopathy of prematurity in zone III with plus disease. The Supplemental Therapeutic Oxygen for Prethreshold Retinopathy of Prematurity (STOP-ROP) study had different criteria for zones I and II threshold retinopathy of prematurity; for zone II disease it used the same definition as the CRYO-ROP study, but in zone I, it defined as 'threshold' any stage of retinopathy of prematurity with plus disease, or stage 3 retinopathy of prematurity with or without plus disease.

\subsection{Prethreshold disease}

The ETROP study however highlighted the importance of the recognition of prethreshold disease and the need for earlier treatment in some babies. This study emphasises the importance of pre plus disease and aggressive zone I and zone II retinopathy of prematurity. Infants screened prior to 30 weeks gestation have hazy corneas and fundoscopy is more difficult. This makes screening prior to 30 weeks difficult and attempting laser treatment is almost impossible. The early onset of threshold disease has been referred to as "rush disease" by some ophthalmologists. It has been reported at 31 to 32 weeks gestational age (Subhani et al. 2001). As a result, the United Kingdom guidelines for screening recommend that the more premature infants should undergo the first screening examination at 30 to 31 weeks post menstrual age.

\section{Early Treatment of Retinopathy of Prematurity (ETROP) Classification:}

Type 1 ROP should be treated:

Zone I any stage of ROP with plus disease

Zone I Stage 3 ROP with or without plus disease

Zone II Stage 2 or 3 ROP with plus disease

Type 2 should be observed, and only undergo treatment if it progresses to type I or threshold disease:

Zone II Stage 1 or 2 ROP without plus disease

Zone II Stage 3 ROP without plus disease

Fig. 1. Classification of treatment guidelines from the Early Treatment of Retinopathy of Prematurity (ETROP) Study.

\subsection{Termination of screening}

Screening for retinopathy of prematurity should continue in accordance with the guidelines until there is no risk of sight threatening disease. The advice in the UK is to continue screening until retinal vascularisation has extended into zone 3 or at 37 weeks in infants in whom no retinopathy of prematurity has developed. In infants who have developed retinopathy of prematurity, screening may cease once regression has been seen on two consecutive examinations. The American guidelines advise ceasing screening when zone 3 or the entire retina is vascularised, the infant is 45 weeks post menstrual age or the retinopathy of prematurity has regressed. 
Screening for retinopathy of prematurity must be safe, cost effective and target those most at risk. Effective screening eliminates unnecessary examinations and reduces wastage of resources and personnel. Retinopathy of prematurity screening requires experience and skill. The ability to recognise severe disease is crucial. All neonatal units should have an agreed policy on screening. Screening includes input from the ophthalmologist, neonatologist and neonatal nurses. Of great importance is the involvement of the neonatal nurses. They play an invaluable role in ensuring infants are included in the screening process at the correct gestational age. They ensure that the pupils are dilated prior to the eye examination and also assist at examinations. The neonatal nurses also play a key role in liaising with both the ophthalmologist and the neonatologist. Screenings should not be delayed or missed as this can result in a poorer visual outcome.

\section{The technique of the screening process}

The screening examinations are usually performed in the neonatal unit with the infant in the incubator and the trained neonatal staff present. The examination requires a well-dilated pupil so that the peripheral retina can be fully visualised. Mydriatic eye drops should be instilled approximately 60 minutes and 30 minutes prior to the examination. A combination of eye drops is preferable to using one single agent. Various combinations of dilating drop regimes exist. Popular combinations include $0.5 \%$ cyclopentolate and $2.5 \%$ phenylephrine or $0.5 \%$ tropicamide and $2.5 \%$ phenylephrine. Most, if not all examinations require a sterile lid speculum. An indenting device may be required in some cases to gently rotate or manipulate the globe.

An indirect ophthalmoscope and a hand held 28 or 30 dioptre convex lens are necessary to perform the retinal examination. The classification scheme for retinopathy of prematurity divides the retina into zones and 12 clock hours and in addition documents the presence or absence of plus or pre plus disease. The examiner should therefore be very familiar with a visual representation of the zones of the retina.

The examiner should first visualise the posterior pole looking for retinopathy of prematurity and in particular note the presence or absence of plus disease. The posterior pole must be scrutinised for flat retinopathy of prematurity and importantly the examiner should be aware that advanced disease in this region may not form a ridge. Failure to recognise this may lead to a delay in treatment and a poorer visual outcome. The nasal and temporal retinal peripheries must then be visualised.

\subsection{Classification of retinopathy of prematurity: zone of retinal involvement}

An important component of the classification of ROP is the zone in which the disease occurs. Zone 1 is a circular area, the radius of which is twice the distance from the optic disc to the fovea (Fig 2). Zone 11 extends concentrically from the edge of zone 1 and its radius extends from the centre of the optic disc to the nasal ora serrata and extends to the equator on the temporal side. Zone 111 consists of the residual temporal crescent of retina (Fig 2).

\subsection{Classification of retinopathy of prematurity: the stage of the disease}

The second component of the classification system is the stage of the disease. Stage 1 retinopathy of prematurity is a thin, flat tortuous, grey-white line that runs approximately parallel with the ora serrata and divides the vascular from the avascular retina (Fig 3). 


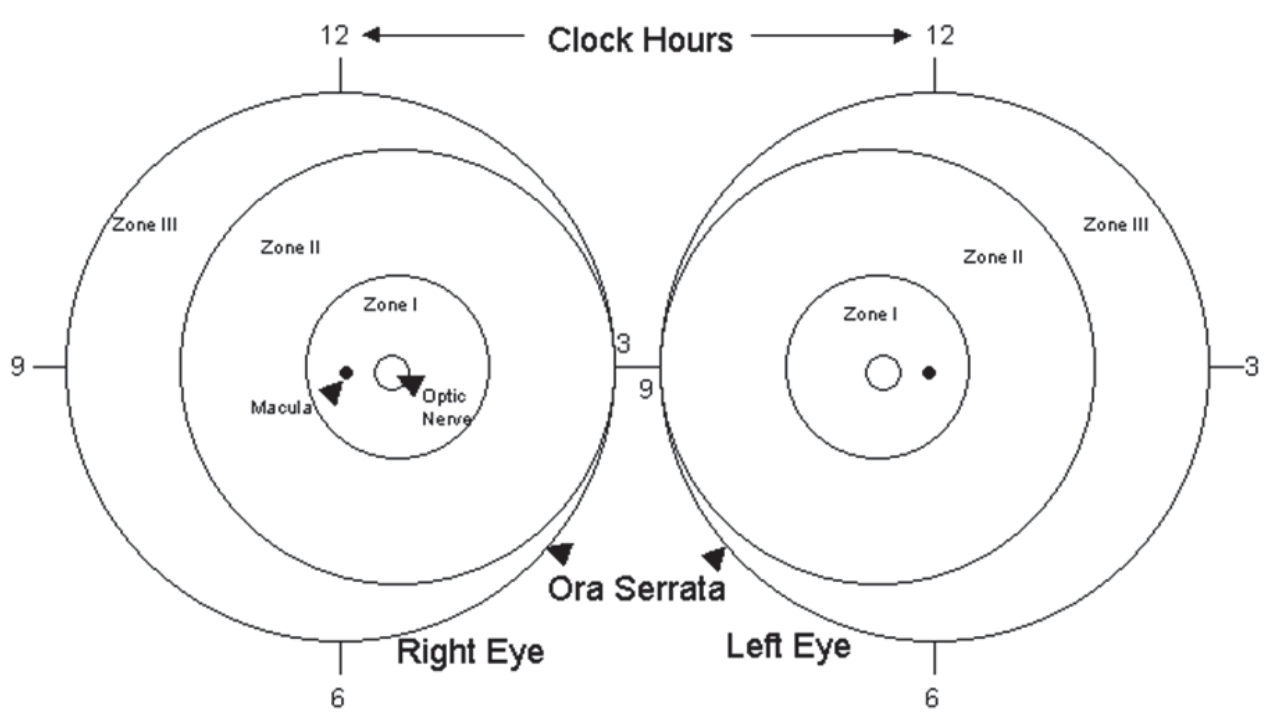

Fig. 2. Zones of the retina and clock hours.

Stage 2 retinopathy of prematurity arises in the region of the line and has height and width and extends above the plane of the retina (Fig 4). Stage 3 signifies extra retinal neovascularisation (Fig 5). The fibrovascular proliferation extends from the ridge into the vitreous, continuous with the posterior aspect of the ridge, causing a ragged appearance as the proliferation becomes more extensive. Stage $4 \mathrm{a}$ indicates extra foveal retinal detachment (Fig 6). Stage $4 \mathrm{~b}$ indicates macular detachment (Fig 7). The detachment is generally concave and circumferentially orientated. In progressive cases the fibrous tissue continues to contract and the detachment increases in height and extends posteriorly and anteriorly. Stage 5 (Fig 8 ) is present when there is a total retinal detachment and it can be an open or closed funnel shape.

\subsection{Plus and pre-plus disease}

The third diagnostic feature is the presence or absence of plus disease which is diagnosed by the presence or absence of vessel dilatation and tortuosity (Fig 9), increasing preretinal and vitreous haemorrhage, vitreous haze and failure of the pupil to dilate. Plus disease signifies a tendency to progression. Pre-plus disease is characterized by abnormal dilatation and tortuosity that is insufficient to be designated as plus disease (Fig 10).

\subsection{Stress-reduction techniques}

The retinopathy of prematurity screening examination process can be distressing for the infant. Distress can be caused by handling the infant in the incubator, inserting the eyelid speculum and use of an indenter to rotate, manipulate or indent the globe. Examinations which extend to more than 2 minutes long are not well tolerated by the infant. There is conflicting evidence as to the physiological changes which occur secondary to the distressing examination. A report by Laws et al. (1996) showed an increase in heart rate of seven beats per minute and a 3\% drop in oxygen saturation after the examination. However, 
Slevin et al. (1997) reported no significant change in heart rate or oxygen saturation but noted that infants expressed distress by their crying patterns and body and limb movements. It was recommended that infants undergoing a screening examination should be placed on a soft padded surface with boundaries that maintained them in a flexed position but allowed for unrestricted movement of the body and limbs. This method of handling may reduce the distress experienced by the infant during the examination process. Other comfort care techniques including administering sucrose solution, nesting, swaddling and/or the use of a pacifier during the screening examination may also be considered. Ophthalmological notes should be made after each screening examination, detailing zone, stage, and extent in terms of clock hours of any retinopathy of prematurity and the presence of any plus or pre-plus disease. These notes should include a recommendation for the timing of the next examination (if any) and be kept with the baby's medical record.

\subsection{Indirect ophthalmoscopy and retcam digital imaging}

Indirect ophthalmoscopy is recognised as the gold standard for retinopathy of prematurity screening. However screening for retinopathy of prematurity with digital imaging has been proposed as a potential alternative. The RetCam 120 (Massie Research Laboratory Inc., Dublin, California) is a digital retinal camera used frequently in paediatric ophthalmology. It is becoming more important for the photo-documentation of retinal conditions in infants with retinopathy of prematurity and retinal haemorrhages associated with "shaken baby syndrome". The hand-held camera is placed gently on the cornea interfaced with ophthalmic lubricant. It produces excellent reproducible images and its advantage over conventional indirect ophthalmoscopy is that of data and image recording and also its ease of use. It is also a very gentle examination in comparison to sclera indentation. However Adams et al. (2004) reported a case of retinal haemorrhaging after RetCam 120 use, highlighting the fragility of the infant retinal vascular system. The RetCam can detect disease up to anterior zone 11 which accounts for over $90 \%$ of retinopathy of prematurity requiring treatment. Despite this, the suitability of the RetCam for screening for retinopathy of prematurity, its sensitivity and specificity for detecting the various stages of the disease remains underdetermined but is gaining more and more acceptance. Yen et al. (2002) concluded that RetCam images had insufficient sensitivity to replace the indirect ophthalmoscope. In contrast, Ells et al. (2003) reported that over the course of a longitudinal series of examinations that digital photography can identify eyes at risk for progression to severe retinopathy of prematurity which would require treatment. Based on a pilot study they reported excellent specificity and sensitivity for remote detection of severe retinopathy of prematurity using the digitised images which were examined remotely by an experienced reader. Wu et al. (2006) compared screening and management results recommended by both indirect ophthalmoscopy and RetCam users and concluded that digital photography had a sensitivity of $100 \%$ and a specificity of $97.5 \%$. In 43 infants they noted that screening and management of retinopathy of prematurity using RetCam imaging did not fail to detect pre-threshold or threshold disease when images could be obtained. However it was noted that indirect ophthalmological examinations were required in $20 \%$ of cases due to poor image quality or overestimation of retinopathy of prematurity. RetCam digital screening has been used with great success in some countries such as Germany. 


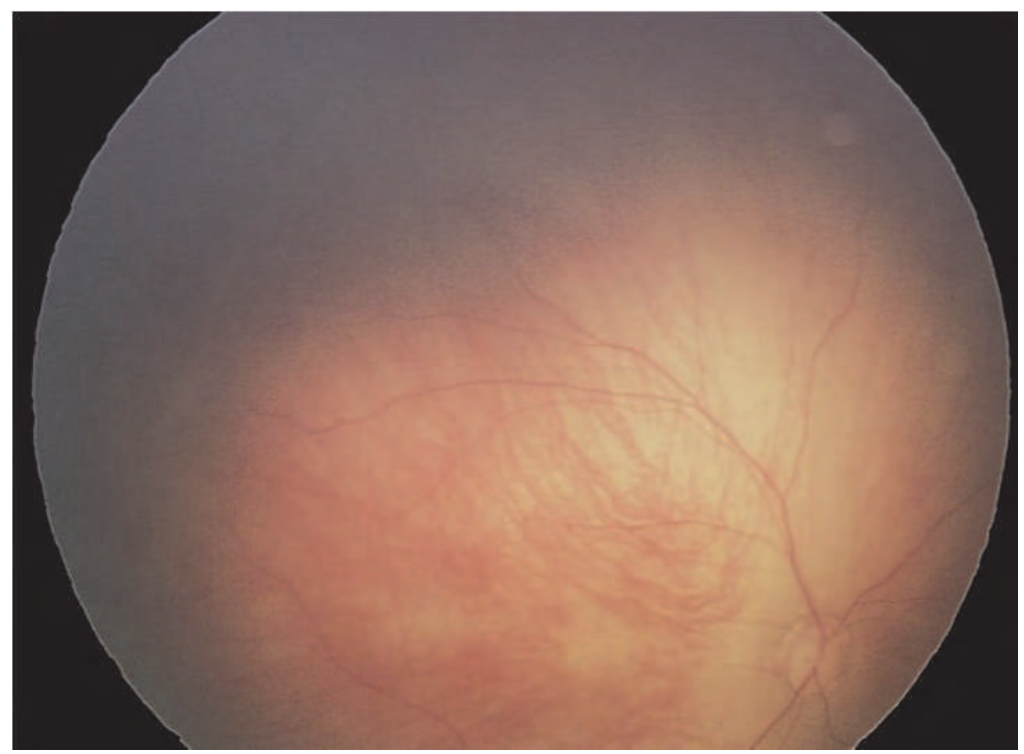

Fig. 3. Stage 1 retinopathy of prematurity. Demarcation line: This is a definite structure that separates the avascular from the vascular retina. 


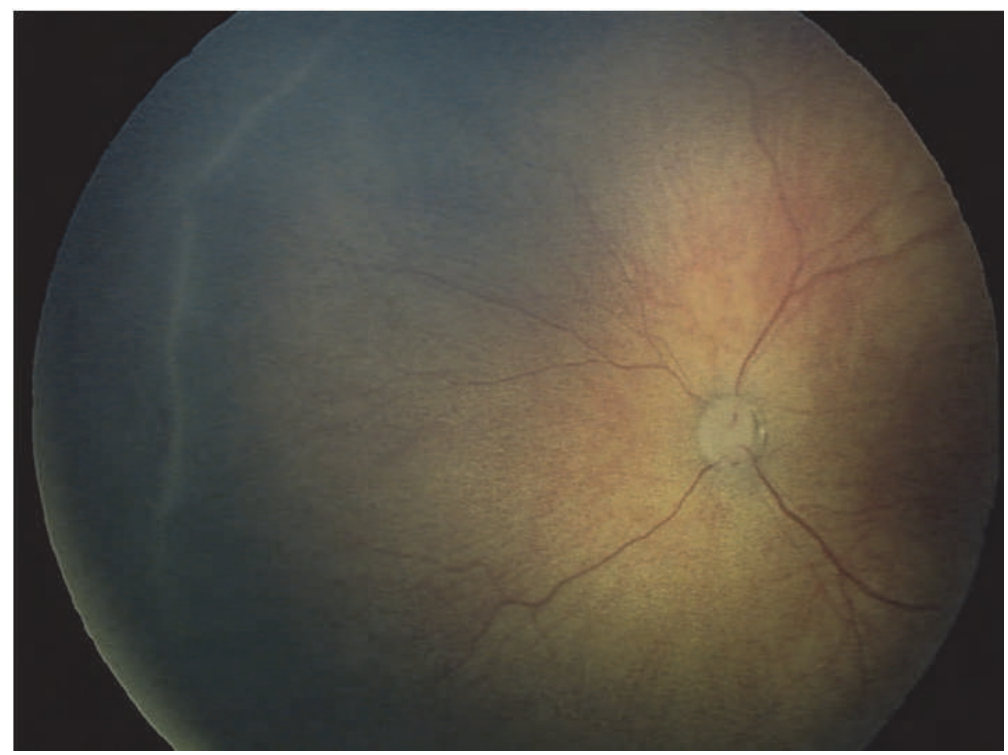

Fig. 4. Stage 2 Retinopathy of Prematurity. The Ridge: This is the hallmark of the stage 2 disease. It arises from the demarcation line and has length and width. It extends above the plane of the retina.

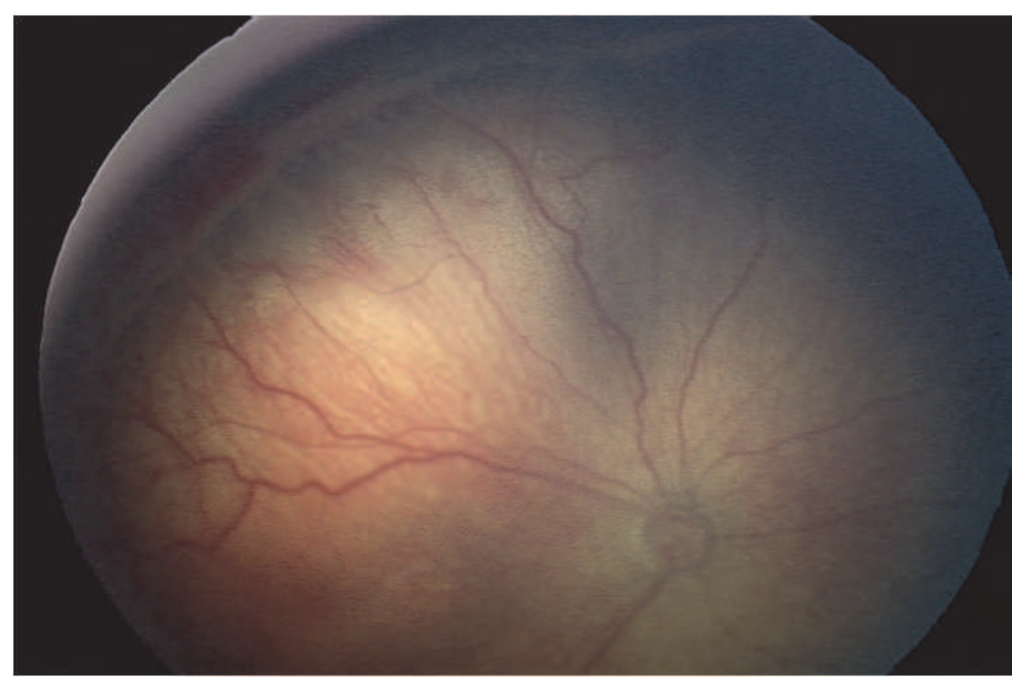

Fig. 5. Stage 3 retinopathy of prematurity. Extra retinal fibrovascular proliferation: Extends from the ridge into the vitreous. It can be divided into mild, moderate and severe depending on vitreous involvement. 


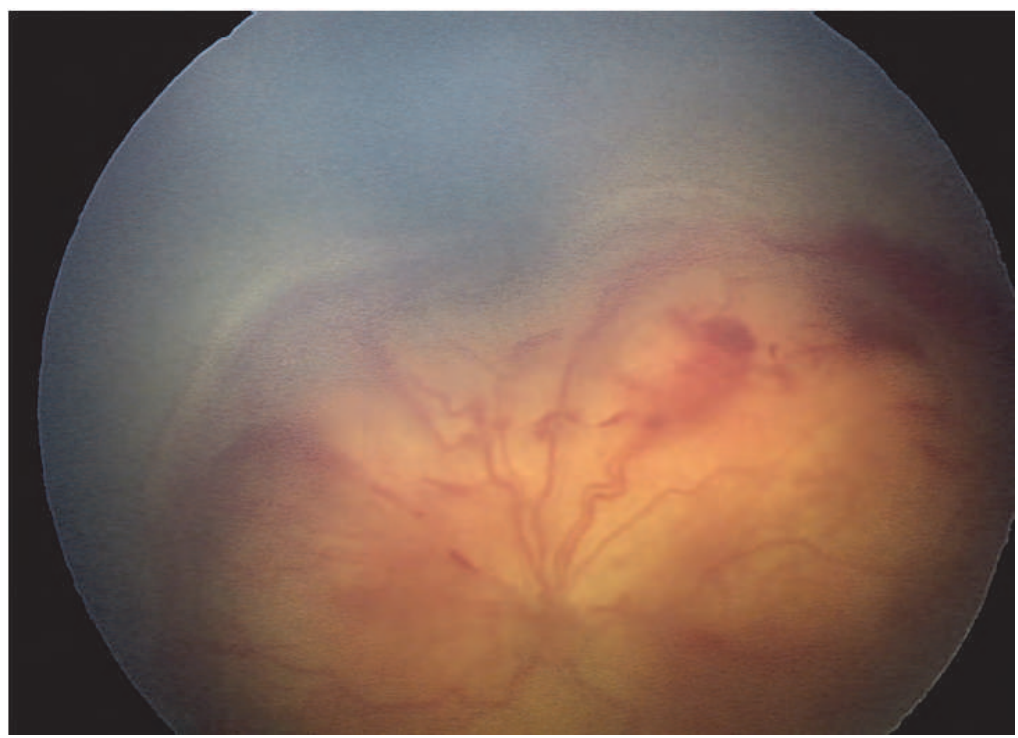

Fig. 6. Stage 4a retinopathy of prematurity: Extra-foveal partial retinal detachment.

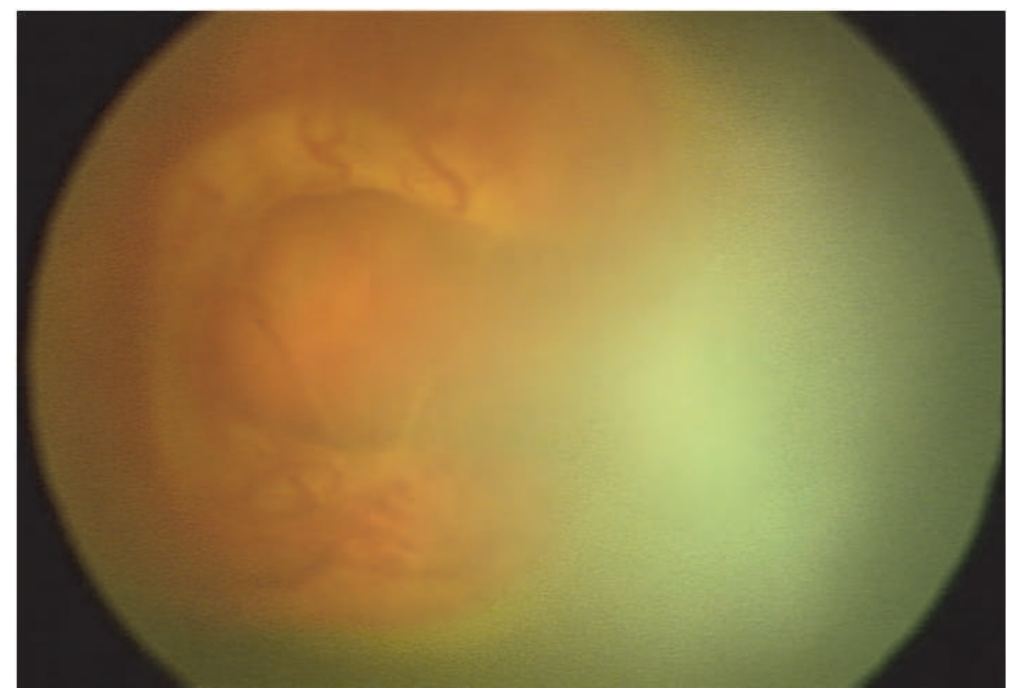

Fig. 7. Stage $4 \mathrm{~b}$ retinopathy of prematurity: Foveal partial retinal detachment. 


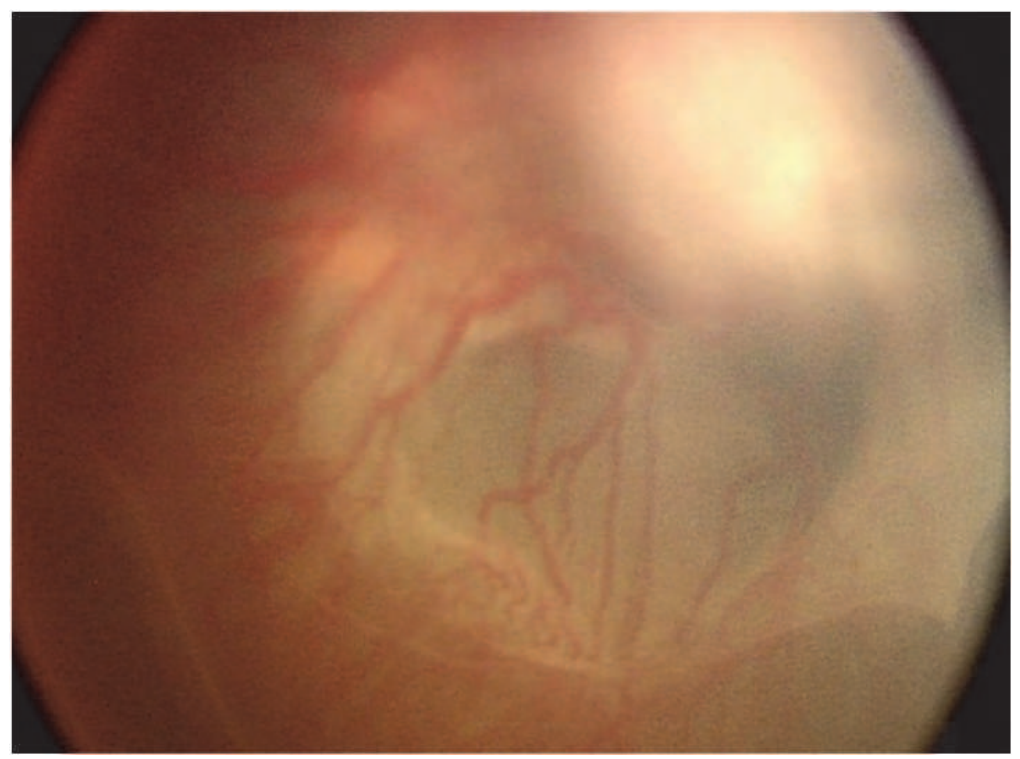

Fig. 8. Stage 5 Retinopathy of prematurity: Total retinal detachment. These detachments are generally tractional and may occasionally be exudative. They are usually funnel shaped.

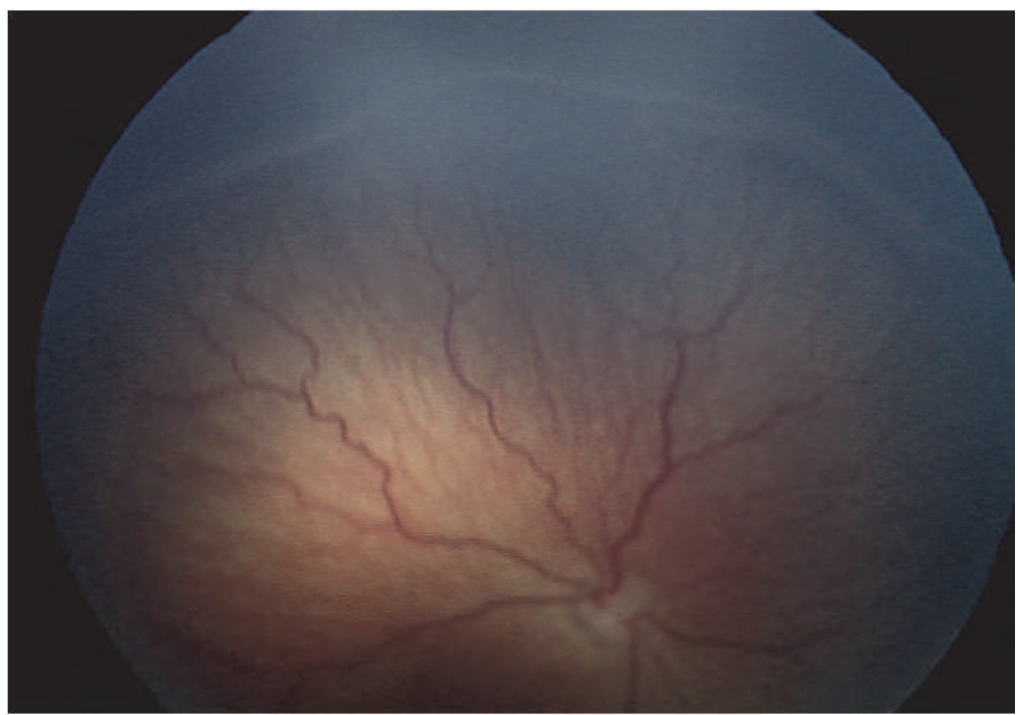

Fig. 9. Plus disease: These are additional signs indicating the severity of active retinopathy of prematurity disease. 1. Venous dilation, anterior tortuosity of posterior pole vessels. 2. Iris vascular engorgement. 3. Pupil rigidity. 4 . Vitreous haze. 


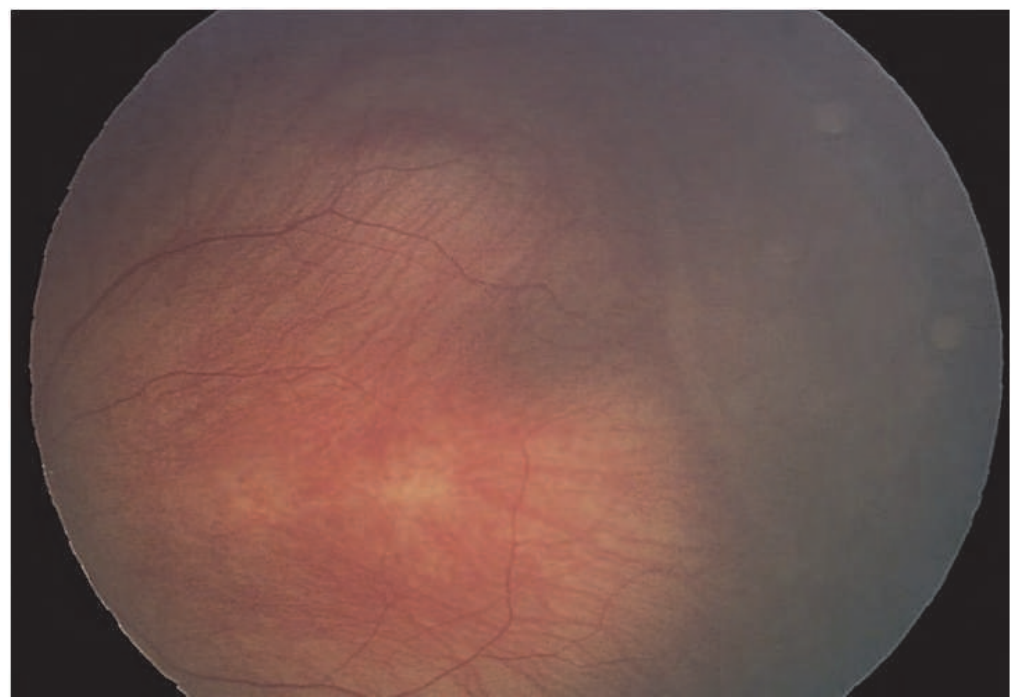

Fig. 10. Pre-plus disease: This is defined as vascular abnormality of the posterior pole but insufficient to form a diagnosis of plus disease but demonstrates more arterial tortuosity and more venous dilation than normal.

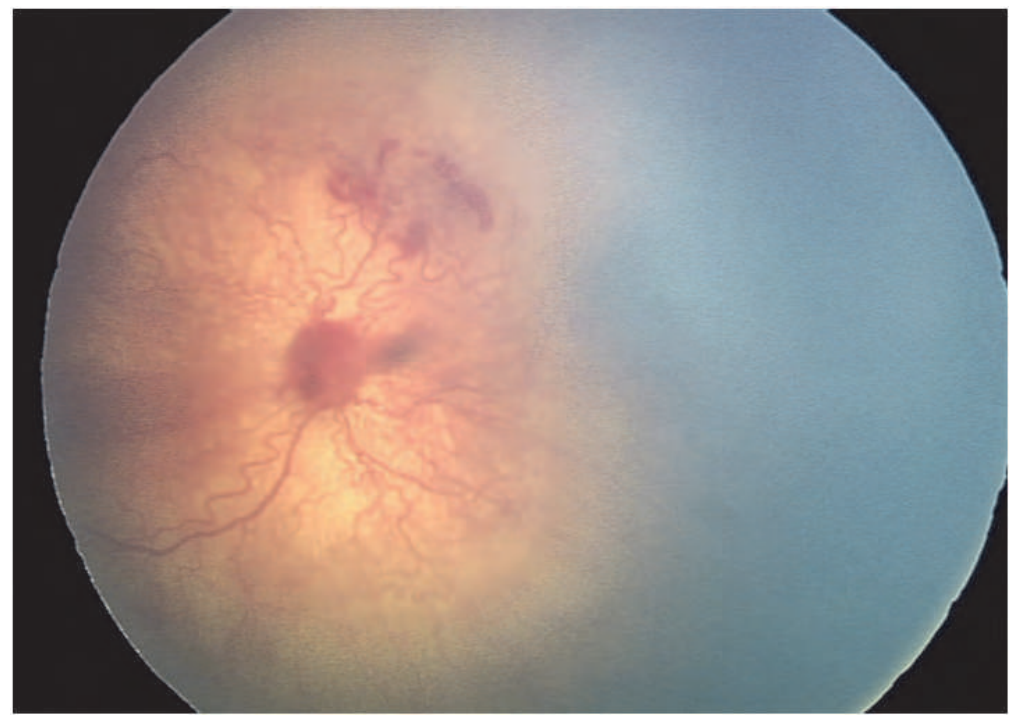

Fig. 11. Aggressive posterior retinopathy of prematurity. This is a rapidly progressive form of retinopathy of prematurity. It can progress to stage 5 retinopathy of prematurity. It has a posterior location with prominence of plus disease. It is observed in zone I or posterior zone II. 


\section{Future advances in screening}

The advent of the telemedicine approach to screening in retinopathy of prematurity raises some important issues. With increasing survivals and expectations for premature infants this is a growing area in terms of litigation. Many ophthalmologists no longer screen for this condition and specialist expertise in retinopathy of prematurity staging is frequently not available in the peripheral hospitals. The reading of digital images by a remote expert could potentially improve the accessibility, quality and cost of retinopathy of prematurity management (Richter et al. 2009). There is the potential for trained neonatal nurses to carry out retinopathy of prematurity screening examinations safely and effectively with the RetCam. This could dramatically reduce the number of indirect ophthalmoscopy examinations needed to be carried out. As a result there would be the potential to maximise effective screening whilst minimising valuable personnel resource time. Diagnostic accuracy and reliability of remote imaging interpretation by experts would have to be assured. Lorenz et al. (2009) reported the success with using wide field digital imaging (RetCam 120) based telemedicine over a six year period where 1,222 infants were screened. They concluded that all treatment requiring retinopathy of prematurity was detected in time with sensitivity for detecting suspected treatment-requiring retinopathy of prematurity at $100 \%$ and a positive predictive value for treatment-requiring retinopathy of prematurity $82.4 \%$. This study is a very positive endorsement for the potential future of telemedicine in retinopathy of prematurity screening. The RetCam is expensive however and may not be cost-effective in those centres where a limited number of infants require screening.

\section{Conclusion}

Screening for retinopathy of prematurity in a timely manner remains the most important initial step in the detection and management of the condition. This is of great importance as it is acknowledged that earlier treatment of the condition results in improved visual outcomes and prognosis. It is becoming increasingly recognized that retinopathy of prematurity is a disease for life and there are significant long-term sequelae. All neonatal units caring for babies at risk of retinopathy of prematurity should have a written protocol in relation to screening. During the screening examination techniques such as nesting the baby will reduce the infants stress and pain and this should be encouraged. Training of ophthalmologists in the technique of screening for retinopathy of prematurity and the recognition of the disease should not be overlooked so that a larger pool of ophthalmologists can perform screening in the peripheral hospitals. Whilst indirect ophthalmoscopy is the gold standard examination device, RetCam digital image systems are becoming increasingly important. The application of telemedicine to retinopathy of prematurity screening is encouraging and positive and in larger neonatal units telemedicine may almost certainly play a much bigger role in the future.

\section{References}

Hunter DG, Mukai S. (1992). Retinopathy of prematurity: pathogenesis, diagnosis and treatment. Int Ophthalmol Clin 1992;32:163-84. 
Gibson DL, Sheps SB,Uh SH, Schechter MT, McCormick AQ.(1990). Retinopathy of prematurity- induced blindness: birth weight-specific survival and the new epidemic. Paediatrics 1990;86:405-12.

Flyn JT, O' Grady GE, Herrera J, Kushner BJ, Cantolino S, Milam W. (1977). Retrolental fibroplasias: clinical observations. Arch Ophthalmol 1977;95:217-23.

An international classification of retinopathy of prematurity. (1984). Paediatrics1984;74(1):127-33.

Multicenter trial of cryotherapy for retinopathy of prematurity. Preliminary results. Cryotherapy for retinopathy of prematurity cooperative group. (1988). Arch Ophthalmol. 1988;106(4):471-479.

Early treatment for retinopathy of prematurity cooperative group. (2003). Revised indications for the treatment of retinopathy of prematurity: results of the early treatment for retinopathy of prematurity randomized trial. Arch Ophthalmol. 2003;121(12):1684-1694.

Gilbert C, Rahi J, Eckstein M, O'Sullivan J, Foster A. (1997).Retinopathy of prematurity in middle-income countries. Lancet. 1997;350(9070):12-14.

Phan MH, Nguyen PN, Reynolds JD.(2003). Incidence and severity of retinopathy of prematurity in Vietnam, a developing middle-income country. J Pediatr Ophthalmol Strabismus. 2003;40(4):208-212.

Screening examination of premature infants for retinopathy of prematurity.(2006).Section on Ophthalmology, American Academy of Pediatrics, American Academy of Ophthalmology American Association for Pediatric Ophthalmology and Strabismus. Pediatrics. 2006;117:572-576.

UK Retinopathy of Prematurity Guideline . Royal College of Paediatrics \& Child Health \& Royal College of Opthalmologists. 2007.

Subhani M, Combs A, Weber P, Gerontis C, DeCristofaro JD. (2011). Screening guidelines for retinopathy of prematurity: the need for revision in extremely low birth weight infants. Pediatrics. 2001;107(4):656-659.

Laws DE, Morton C, Weindling M, Clark D. (1996). Systemic effects of screening for retinopathy of prematurity. Br J Ophthalmol. 1996;80(5):425-428.

Slevin M, Murphy JF, Daly L, O'Keefe M.(1997). Retinopathy of prematurity screening, stress related responses, the role of nesting. Br J Ophthalmol. 1997;81(9):762-764.

Adams GW, Clark BJ, Fang S, Hill S.(2004). Retinal haemorrhages in an infant following RetCam screening for retinopathy of prematurity. Eye. 2004;18:652-653.

Yen KG, Hess D, Burke B, Johnson RA, Feuer WJ, Flynn JT. (2002). Telephotoscreening to detect retinopathy of prematurity: preliminary study of the optimum time to employ digital fundus camera imaging to detect ROP. J AAPOS. 2002;6(2):64-70.

Ells AL, Holmes JM, Astle WF, Williams G, Leske DA, Fielden M, et al. (2003).Telemedicine approach to screening for severe retinopathy of prematurity: a pilot study. Ophthalmology. 2003;110(11):2113-2117.

Wu C, Petersen RA, VanderVeen DK. (2006). RetCam imaging for retinopathy of prematurity screening. J APPOS. $2006 ; 10(2): 107-11$.

Richter GM, Williams SL, Sarren J, Flynn JT, Chiang MF. (2009). Telemedicine for retinopathy of prematurity diagnosis: evaluation and challenges. Surv Ophthalmol.2009;54(6):67-85. 
Lorenz B, Spasovska K, Elflein H,Schneider N. (2009). Wide-field digital imaging based telemedicine for screening for acute retinopathy of prematurity (ROP). Six-year results of a multicentre field study. Graefes Arch Clin Exp Ophthalmol. 2009;247(9):1251-62. 


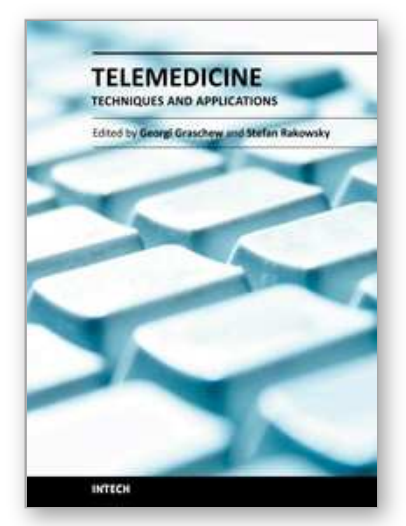

\author{
Telemedicine Techniques and Applications \\ Edited by Prof. Georgi Graschew
}

ISBN 978-953-307-354-5

Hard cover, 514 pages

Publisher InTech

Published online 20, June, 2011

Published in print edition June, 2011

Telemedicine is a rapidly evolving field as new technologies are implemented for example for the development of wireless sensors, quality data transmission. Using the Internet applications such as counseling, clinical consultation support and home care monitoring and management are more and more realized, which improves access to high level medical care in underserved areas. The 23 chapters of this book present manifold examples of telemedicine treating both theoretical and practical foundations and application scenarios.

\title{
How to reference
}

In order to correctly reference this scholarly work, feel free to copy and paste the following:

Claire Hartnett and Michael O'Keefe (2011). Screening for Retinopathy of Prematurity, Telemedicine Techniques and Applications, Prof. Georgi Graschew (Ed.), ISBN: 978-953-307-354-5, InTech, Available from: http://www.intechopen.com/books/telemedicine-techniques-and-applications/screening-for-retinopathy-ofprematurity

\section{INTECH}

open science | open minds

\section{InTech Europe}

University Campus STeP Ri

Slavka Krautzeka 83/A

51000 Rijeka, Croatia

Phone: +385 (51) 770447

Fax: +385 (51) 686166

www.intechopen.com

\section{InTech China}

Unit 405, Office Block, Hotel Equatorial Shanghai

No.65, Yan An Road (West), Shanghai, 200040, China

中国上海市延安西路65号上海国际贵都大饭店办公楼405单元

Phone: +86-21-62489820

Fax: $+86-21-62489821$ 
(C) 2011 The Author(s). Licensee IntechOpen. This chapter is distributed under the terms of the Creative Commons Attribution-NonCommercialShareAlike-3.0 License, which permits use, distribution and reproduction for non-commercial purposes, provided the original is properly cited and derivative works building on this content are distributed under the same license. 